\title{
gs \\ Double stop-band structure near half-integer tunes in high-intensity rings
}

\author{
K. Moriya, M. Ota, K. Fukushima, ${ }^{*}$ M. Yamaguchi, K. Ito, and H. Okamoto ${ }^{\dagger}$ \\ Graduate School of Advanced Sciences of Matter, Hiroshima University, \\ 1-3-1 Kagamiyama, Higashi-Hiroshima 739-8530, Japan \\ (Received 16 May 2016; published 2 November 2016)
}

\begin{abstract}
This paper addresses a detailed experimental study of collective instability bands generated near every half-integer tune per lattice period by coherent dipole and quadrupole resonances. Both instabilities appear side by side or overlap each other but are mostly separable because the dipole resonance often creates a narrower stop band accompanied by more severe particle losses. The separation of these low-order resonance bands becomes greater as the beam intensity increases. In principle, the double stop-band structure can be formed even without machine imperfections when the beam's initial phase-space profile is deviated from the ideal stationary distribution. The tabletop ion-trap system called "S-POD" is employed to experimentally demonstrate the parameter dependence of the double stop-band structure. Numerical simulations are also performed for comparison with experimental observations.
\end{abstract}

DOI: 10.1103/PhysRevAccelBeams.19.114201

\section{INTRODUCTION}

An intense hadron beam exhibits complex collective behavior due to the strong Coulomb coupling among individual charged particles. The whole beam acts like a single organized object rather than a group of many particles. The collective motion can be decomposed into an infinite number of modes whose oscillation frequencies depend on the beam density. A self-consistent analytic treatment of such coherent modes was first developed by Sacherer who concluded that the beam becomes unstable when the tune of a particular mode agrees with that of a Fourier component in the external periodic driving force [1]. His betatron resonance condition reads $\Omega_{m}=n$ where $\Omega_{m}$ is the tune of the coherent mode of order $m$, and $n$ is a positive integer. When the bare tune of a high-intensity ring is $\nu_{0}$ in either the horizontal or vertical direction, $\Omega_{m}$ can approximately be expressed as $[1,2]$

$$
\Omega_{m}=m\left(\nu_{0}-C_{m} \Delta \nu\right),
$$

where $C_{m}$ is a positive constant depending on the mode number $m$, and $\Delta \nu$ stands for the tune shift induced by the collective Coulomb potential [3]. Sacherer pointed out that $C_{m}$ is smaller than unity for all coherent modes. We here follow the notation adopted in past papers $[1,2]$. The mode number $m$ corresponds to the order of the transverse driving potential. The $m=2$ mode has elliptical symmetry in

\footnotetext{
*Present address: Facility for Rare Isotope Beams, Michigan State University, East Lansing, MI 48824, USA.

tokamoto@sci.hiroshima-u.ac.jp

Published by the American Physical Society under the terms of the Creative Commons Attribution 3.0 License. Further distribution of this work must maintain attribution to the author $(s)$ and the published article's title, journal citation, and DOI.
}

phase space and can be driven by the linear potential proportional to the square of a transverse coordinate. The third-order driving term can excite the sextupole resonance of the $m=3$ mode, the fourth-order octupole term directly drives the $m=4$ mode, and so on.

For a circular machine consisting of $N_{\mathrm{sp}}$ superperiodic structures, the Fourier expansion of the external driving force around the ring has only every $N_{\text {sp }}$ harmonics. The resonance condition then takes the form

$$
\Omega_{m}=N_{\mathrm{sp}} \cdot n,
$$

instead of $\Omega_{m}=n$. Strictly speaking, any machine includes finite mechanical imperfections that give rise to all Fourier harmonics $\left(N_{\mathrm{sp}} \rightarrow 1\right)$, but these error-induced harmonics are generally much weaker than the original structureinduced harmonics. We, therefore, simply ignore them in the following study. At low beam intensity where $\Delta \nu \approx 0$, Eq. (2) is reduced to the well-known single-particle resonance condition $\nu_{0} \approx N_{\mathrm{sp}} \cdot n / m$. It is now clear that the instability of the quadrupole mode $(m=2)$ occurs near half-integer tunes, as emphasized in standard textbooks. Equation (2) also says that the dipole mode $(m=1)$ becomes unstable at integer tunes.

Most Vlasov theories including Sacherer's have relied on the smooth approximation that considerably simplifies the mathematical procedure to solve the basic equations. The approximation, however, kills the instability of interest; no resonance takes place in a uniform focusing channel. A sort of mathematical trick is thus required to derive the instability condition like Eq. (2) under the smooth approximation. Specifically, a periodic perturbing force is introduced later to excite resonance forcibly. Such a trick, of course, becomes unnecessary if we quit the lattice smoothing and just keep the periodic nature of alternating gradient (AG) beam transport in the Vlasov analysis. Incorporating 
an AG lattice explicitly into the theory, we find a variety of coherent resonances to occur under the condition

$$
\Omega_{m}=N_{\mathrm{sp}} \cdot \frac{n}{2},
$$

even if the external driving force is perfectly linear [4]. This type of resonance instability was first numerically identified in connection with high-intensity linear transport channels where $N_{\mathrm{sp}}=1$ [5-7].

According to the Vlasov theory in Ref. [4], the growth rate and stop-band width of self-field-driven resonance under the condition (3) are proportional to the beam perveance, which means that this instability becomes less important as the beam density decreases. At low beam intensity, we only need to care about external-field-driven resonance under condition (2) [8,9]. Equation (3) suggests that, in a high-intensity ring, there are twice as many resonance stop bands as expected from the popular condition in Eq. (2). We have already shown experimental evidence that there should be not only the fourth-order $(m=4)$ nonlinear resonance but also the self-field-driven linear $(m=2)$ resonance overlapping at quarter-integer tunes or, in other words, at the betatron phase advance of 90 degrees per lattice period [10-14]. Even the existence of the third-order $(m=3)$ resonance has been confirmed at the phase advance of 60 degrees [10-14].

We now focus on the lowest-order resonance, i.e., the instability of the dipole mode $(m=1)$. Equation (3) leads to the dipole resonance condition

$$
\nu_{0}=N_{\mathrm{sp}} \cdot \frac{n}{2},
$$

because $C_{1}=0$ [15]. This condition can readily be understood if we regard the whole beam as a rigid body. The coherent dipole instability is closely related to the halfinteger resonance of a single macroparticle exposed to a periodic linear force. The basic feature can thus be well explained by Hill's equation. On the other hand, a similar condition is derived from Eq. (2) for the quadrupole mode $(m=2)$. Substitution of $m=2$ into Eq. (2) gives

$$
\nu_{0}-C_{2} \Delta \nu=N_{\mathrm{sp}} \cdot \frac{n}{2},
$$

which approaches Eq. (4) as the tune shift $\Delta \nu$ gets smaller. The same condition is concluded also from Eq. (3) for even harmonics. Equations (4) and (5) indicate that there could be two essentially different types of low-order resonances near half-integer tunes.

In the present paper, we experimentally demonstrate the existence of these two half-integer instabilities by using a novel tabletop system "S-POD" (Simulator of Particle Orbit Dynamics) at Hiroshima University. After a brief description of S-POD in Sec. II, various experimental results obtained with this unique apparatus are given in Sec. III. We show that a relatively wide stop band of the quadrupole resonance is accompanied by a very narrow dipoleresonance band near every half-integer tune per lattice superperiod. Experimental observations are then compared with particle-in-cell (PIC) simulations in Sec. IV. Concluding remarks are finally made in Sec. V.

\section{S-POD}

S-POD is a compact plasma-trap facility designed solely for beam dynamics studies $[10,14,16,17]$. The idea is based on the fact that the collective motion of a non-neutral plasma confined in a linear Paul trap (LPT) can be made almost equivalent to that of a space-charge-dominated beam traveling through an AG focusing channel [18]. Detailed information of S-POD has been given in many previous publications [10-14], so we here only briefly explain its essence for later convenience.

The equation of the transverse motion of ions in a LPT can be written as

$$
\frac{d^{2} x}{d t^{2}}+K(t) x=-I \frac{\partial \phi_{\mathrm{sc}}}{\partial x}
$$

where $x$ is the transverse spatial coordinate of a stored ion, $I$ is a constant depending on ion species, $K(t)$ is a periodic function with respect to the independent variable $t$ (time), and $\phi_{\mathrm{sc}}$ is the collective Coulomb potential satisfying the Poisson equation. A similar equation holds for the transverse $y$-direction perpendicular to the $x$-axis. Equation (6) is identical to the well-known betatron equation frequently adopted as a starting point in past theoretical studies of intense hadron beam dynamics where the self-field potential $\phi_{\text {sc }}$ plays a crucial role $[1,2,4-8]$. This fact indicates that we can approximately reproduce the collective motion of a space-charge-dominated beam in a large-scale machine within a compact tabletop apparatus [18].

Figure 1 is a picture of the multisectioned LPT used for the present study. Four electrode rods are symmetrically placed around the axis to provide a radio-frequency (rf)

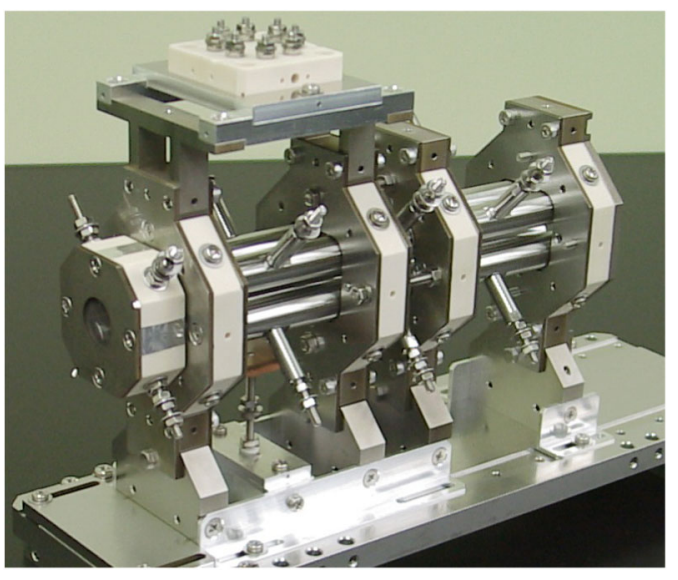

FIG. 1. A typical linear Paul trap for S-POD. 

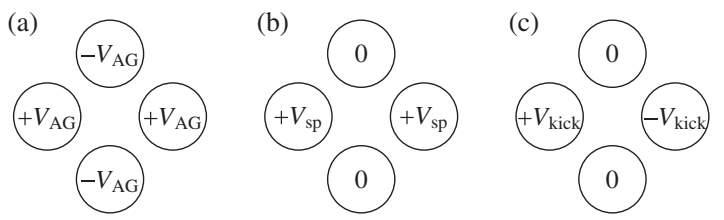

FIG. 2. Electrode excitation patterns employed for the present experiment. The four circles in each picture represent the cross sections of the quadrupole electrodes aligned along the LPT axis. (a) Quadrupole excitation for transverse ion confinement. The periodic voltage $V_{\mathrm{AG}}(t)$ generates a strong AG focusing force. (b) Superperiodic pulse excitation. $V_{\mathrm{sp}}$ is superimposed on $V_{\mathrm{AG}}$ in order to introduce a specific superperiodic modulation to the focusing function $K(t)$. (c) Horizontal dipole excitation. A pulse voltage $V_{\text {kick }}$ is switched on once at the beginning, if necessary, to kick an ion bunch horizontally.

quadrupole potential [19]. In order to confine a large number of ions within a narrow space surrounded by the quadrupole rods, we apply time-dependent voltages $\pm V_{\mathrm{AG}}(t)$ as shown in Fig. 2(a). In a regular LPT, the time variation of $V_{\mathrm{AG}}(t)$ is mostly sinusoidal. The aperture size is $1 \mathrm{~cm}$ in diameter and the overall length of the LPT is roughly $20 \mathrm{~cm}$. We usually trap ${ }^{40} \mathrm{Ar}^{+}$ions produced from neutral Ar gas atoms via the electron bombardment process. The species of stored ions does not matter because it is one of several scaling parameters to adjust fundamental beamdynamics quantities such as the bare tune $\nu_{0}$, the tune shift $\Delta \nu$, etc. Needless to say, the cost of a LPT is incomparably lower than any accelerators. We have constructed more than five LPTs of different designs optimized for different experimental purposes. Another LPT system, substantially the same as S-POD, is under construction at Rutherford Appleton Laboratory [20]. Gilson and his co-workers of Princeton Plasma Physics Laboratory also developed a relatively large LPT dedicated to beam physics [21-23].

S-POD clearly has many practical advantages other than the economical aspect. Most importantly, the focusing function $K(t)$ can very easily be controlled over a wide range. Since $K(t)$ is proportional to the voltages applied to the quadrupole electrodes, all we need for the study of lattice-dependent effects is to change the rf waveform from the power supply. No mechanical modification to the LPT is necessary. In contrast, any particle accelerator has a unique uncontrollable $K(t)$, which prevents one from exploring how beam stability depends on diverse designs of AG lattices. S-POD provides us with a simple experimental environment free from various unwanted noise sources inevitable in large-scale machines. It is thus possible to clarify the physics that obeys the very basic model in Eq. (6). This also means, however, that some complex beam dynamic effects are out of the scope of the present study. For instance, the dispersive effect originating from bending magnets is missing in our model.

The reproducibility of S-POD data is very good. It is partly due to the simpleness and stability of the whole system.
Another important factor is the availability of an ion plasma well matched to the external rf potential. We usually spend a second, corresponding to a million AG periods, to accumulate ions slowly through the electron bombardment process. The final number of ions can be controlled by changing the neutral Ar gas pressure and/or the current of the electron beam from an $e$-gun. After an ion bunch is formed at a certain stable operating point, we shut down the electron beam, wait typically for $50 \mathrm{~ms}$ until the bunch settles into a quasistationary state, and finally move the operating point to a specific position of interest for stability measurement. This elaborate procedure enables us to start each measurement cycle with a well-conditioned equilibrium plasma. See the experimental results in the next section. For example, each curve in Figs. 4 and 5 consists of nearly 1,000 independent data points obtained through 1,000 independent measurements. Nevertheless, it is very smooth over a wide tune range. We can reproduce almost identical stop-band distributions acquiring essentially the same data at any time [10-14]. Unlike very time-consuming PIC simulations, it takes us just 10 seconds or shorter to complete a single measurement cycle in S-POD. Furthermore, we do not have to stay beside the S-POD control system to retune experimental parameters because everything is completely automated. Only less than 3 hours is enough to store 1,000 measurement data in a personal computer.

\section{EXPERIMENT}

An extremely wide range of choices is available for the waveform of the focusing function $K(t)$. We here separate $K(t)$ into two parts as $K(t)=K_{\mathrm{AG}}(t)+\Delta K(t) . K_{\mathrm{AG}}(t)$ is the primary AG focusing function proportional to $V_{\mathrm{AG}}(t)$ in Fig. 2(a). For the sake of simplicity, we take the sinusoidal focusing model in which a single FODO waveform is

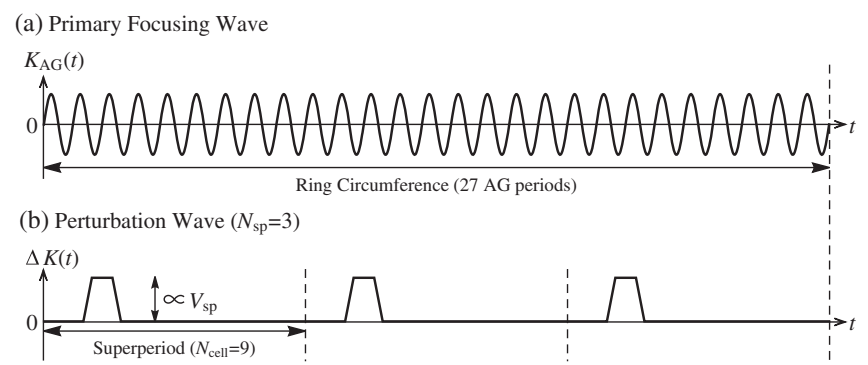

FIG. 3. Radio-frequency waveforms employed for the present experiment. We have assumed an AG lattice consisting of three superperiodic structures $\left(N_{\mathrm{sp}}=3\right)$, each of which includes nine unit focusing cells $\left(N_{\text {cell }}=9\right)$. The discrete FODO waveform is approximated by a sine curve for simplicity. The upper panel (a) thus emulates an AG beam transport channel composed of 27 identical FODO cells. We superimpose the periodic pulses in the lower panel (b) to generate superperiodic driving harmonics in the focusing function $K(t)$ in Eq. (6). Note that the superperiodic number $N_{\mathrm{sp}}$ is of no physical importance as long as we disregard lattice imperfections. 
approximated by a sine curve; namely, $K_{\mathrm{AG}}(t)$ is a sinusoidal function with respect to time $t$ as illustrated in Fig. 3(a). According to past S-POD experiments [10-14], this simplification does not affect the fundamental feature of coherent resonance. The second part $\Delta K(t)$, generated by a low stepwise voltage $V_{\text {sp }}$ in Fig. 2(b), is a linear perturbation that allows us to introduce an arbitrary superperiodic modulation to the function $K(t)$. We switch on $V_{\text {sp }}$ every $N_{\text {cell }}$ AG periods defined by the primary focusing wave of $K_{\mathrm{AG}}(t)$. The pattern (b) in Fig. 2 is nothing but a quadrupole excitation. It is clearly equivalent to the pattern (a) with $V_{\mathrm{AG}}$ replaced by $V_{\mathrm{sp}} / 2$. If $V_{\mathrm{sp}}$ is positive, this electrode excitation gives horizontal focusing and vertical defocusing forces to positively charged ions. In the present experiment, the ratio of the perturbing quadrupole amplitude $V_{\mathrm{sp}} / 2$ to the sinusoidal focusing amplitude $\max \left(V_{\mathrm{AG}}\right)$, denoted by $\alpha \equiv V_{\mathrm{sp}} / 2 \max \left(V_{\mathrm{AG}}\right)$, is set in a range from zero to a few percent.

Apart from the periodic voltages $V_{\mathrm{AG}}$ and $V_{\mathrm{sp}}$, a single pulse perturbation in Fig. 2(c) is applied to the plasma only initially when needed. The voltages $\pm V_{\text {kick }}$ on the horizontal pair of the electrodes produce a dipole field in the LPT aperture. This electrode excitation pattern is used later (see Sec. III C) to activate the coherent dipole motion by artificially displacing the plasma centroid from the LPT axis at the beginning.

The sample waveforms for $K_{\mathrm{AG}}(t)$ and $\Delta K(t)$ in Fig. 3 emulate a circular machine with $N_{\text {cell }}=9$ and $N_{\mathrm{sp}}=3$. This combination of $N_{\text {cell }}$ and $N_{\text {sp }}$ reflects the lattice condition of the rapid cycling synchrotron (RCS) at the Japan Proton Accelerator Research Complex (J-PARC) [24]. The RCS is composed of three lattice superperiods $\left(N_{\mathrm{sp}}=3\right)$, each of which includes nine AG focusing cells $\left(N_{\text {cell }}=9\right)$. The superposition of the two periodic waves in Fig. 3 yields driving Fourier harmonics that are more or less similar to the RCS situation. We, however, immediately understand from Fig. 3 that the superperiodic number $N_{\mathrm{sp}}$ is inessential here. All information necessary for a complete description of beam resonance is contained in a single superperiod as long as the machine holds perfect $N_{\mathrm{sp}}$-fold symmetry. We are allowed to pay attention only to a lattice superperiod instead of looking at the whole ring. It is then convenient to use the bare tune per lattice period, i.e., $Q_{0}\left(\equiv \nu_{0} / N_{\mathrm{sp}}\right)$, rather than the one-turn tune $\nu_{0} . \Delta \nu$ should also be replaced by the tune shift per lattice period, $\Delta Q\left(\equiv \Delta \nu / N_{\mathrm{sp}}\right)$. The resonance conditions (2) and (3) are now free from the superperiodicity, resulting in

$m\left(Q_{0}-C_{m} \Delta Q\right)= \begin{cases}n & (\text { external-field-driven }) \\ n / 2 & (\text { self-field-driven })\end{cases}$

If the superperiod has approximate $P$-fold symmetry, both resonances will particularly be enhanced with the harmonic numbers of $n=P, 2 P, 3 P, \ldots$
Throughout this paper, we assume $N_{\text {cell }}=9$ as sketched in Fig. 3, but the basic physics never changes for other choices of $N_{\text {cell }}$. We have already tried another example with $N_{\text {cell }}=5$ (and $N_{\text {sp }}=10$ ), bearing in mind the Proton Synchrotron at CERN [25]. The result was essentially the same as described in the subsections below.

The frequency of the sinusoidal focusing field in Fig. 3(a) is chosen to be $1 \mathrm{MHz}$. Then, the maximum rf voltage required for a full tune-space survey is less than about $100 \mathrm{~V}$ for ${ }^{40} \mathrm{Ar}^{+}$ions. The full width at half maximum (FWHM) of each perturbing pulse in Fig. 3(b) is fixed at $1 \mu \mathrm{s}$ while the pulse height $V_{\mathrm{sp}}$ is varied as a free parameter. The rise time and fall time of the pulse are set at $0.3 \mu \mathrm{s}$. Although the horizontal and vertical bare tunes $\left(Q_{0 x}, Q_{0 y}\right)$ can independently be controlled if necessary, we set them equal, i.e., $Q_{0 x}=Q_{0 y}\left(\equiv Q_{0}\right)$, in the absence of the perturbing pulses. This setup results in a slight discrepancy between the horizontal and vertical net focusing forces whenever the perturbing pulses are excited. The effect of this tune discrepancy on stop bands has been observed experimentally and numerically, which shall be discussed below in detail. We confine a large number of ${ }^{40} \mathrm{Ar}^{+}$ions in the LPT typically for 1-10 ms corresponding to 37-370 turns around the model ring with $N_{\text {sp }}=3$ and $N_{\text {cell }}=9$. After a specific storage period at a specific working point, stored ions are extracted from the LPT toward either a Faraday-cup detector or a microchannel plate (MCP) with a phosphor screen.

\section{A. Primary stop bands in the absence of the superperiodic perturbation}

Since each superperiod of the model ring in Fig. 3 contains nine AG cells, the bare tune $Q_{0}$ ranges from 0 to $4.5(=0.5 \times 9)$. In the absence of the perturbing pulses in Fig. 3(b), the lattice superperiod possesses perfect ninefold symmetry $(P=9)$. Low-order self-field-driven resonances are then expected to occur under the condition

$$
Q_{0} \approx \frac{9}{2}\left(\frac{n}{m}\right)+C_{m} \Delta Q
$$

Equation (8) predicts possible ion losses at $Q_{0} \approx 2.25$ ( $m=2$ : quadrupole), $Q_{0} \approx 1.5$ and 3 ( $m=3$ : sextupole), $Q_{0} \approx 1.125,2.25$, and 3.375 ( $m=4$ : octupole), provided that the second term on the right-hand side is small. The bare tune $Q_{0}$ is related to the betatron phase advance $\sigma_{0}$ per single $\mathrm{AG}$ cell as $\sigma_{0}=360^{\circ} \times\left(Q_{0} / P\right) . Q_{0}$ of $1.125,1.5$, $2.25,3$, and 3.375 thus correspond, respectively, to $\sigma_{0}$ of $45^{\circ}, 60^{\circ}, 90^{\circ}, 120^{\circ}$, and $135^{\circ}$. The coherent dipole stop band $(m=1)$ is located at the edge of the stability domain $\left(Q_{0}=4.5\right)$ and thus undetectable. The number of ${ }^{40} \mathrm{Ar}^{+}$ ions surviving after a 10-ms storage in the LPT without the superperiodic perturbation is plotted in Fig. 4 as a function of $Q_{0}$. We initially stored around $10^{5}, 10^{6}$, and $10^{7}$ ions to 


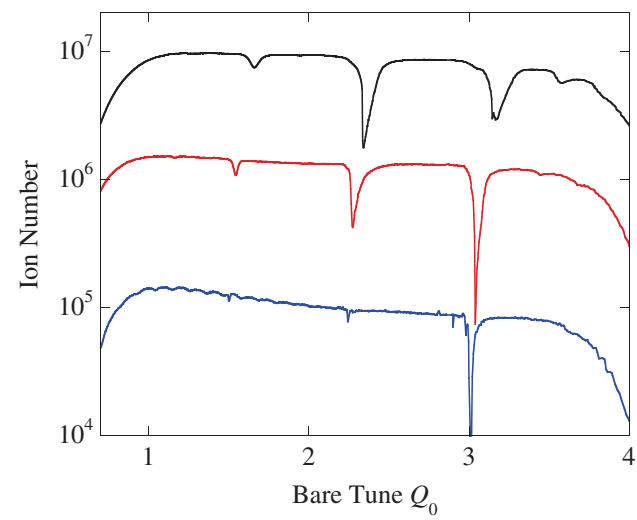

FIG. 4. Stop-band distribution in the absence of the superperiodic perturbation. The number of ${ }^{40} \mathrm{Ar}^{+}$ions in the LPT is measured with the MCP after a storage period of $10 \mathrm{~ms}$. The horizontal and vertical bare tunes have been set equal, i.e., $Q_{0 x}=Q_{0 y}\left(\equiv Q_{0}\right)$, in all measurements carried out over the range $2 / 3 \leq Q_{0} \leq 4$. Three different initial ion numbers, i.e., approximately $10^{5}$ (blue), $10^{6}$ (red), and $10^{7}$ (black), are considered in this example. Rapid drops in ion number near both ends of the abscissa are simply because the operating point is too close to the stability boundary of Hill's equation. Each curve is composed of 979 discrete data points obtained at 979 different bare tune values.

figure out the intensity dependence of stop-band locations. Three clear instability regions are identified at bare tunes slightly above $1.5,2.25$, and 3 related, respectively, to the sextupole, quadrupole (or octupole), and sextupole resonances [10-14]. There is a possibility of higher-order nonlinear resonances overlapping at these tunes, but the observed ion losses should be due mainly to a few loworder resonances. Owing to the space-charge-induced tune shift $\Delta Q$, all these stop bands move to the higher tune side as the plasma density is increased. We can make a rough estimate of the tune depression, i.e., the ratio of the spacecharge-depressed effective tune to the bare tune, by measuring the shift of a major stop band from its original position at zero intensity. According to a previous experimental estimate based on a similar LPT, the tune depression reaches $0.85-0.9$ at the stored ion number of $10^{7}$ [12]; namely, the space-charge-induced tune shift exceeds $10 \%$ of the bare tune, which should be beyond the maximum tune shift achievable in an ordinary circular machine.

It is evident from Fig. 4 that the instabilities at $Q_{0} \approx 1.5$ and 2.25 become more prominent at higher plasma intensity. This strongly suggests that the self-field potential is responsible for the excitation of these two resonances. In particular, ion losses near $Q_{0}=2.25$ have become most severe at high density, which convinces us that the primary cause of this instability should be the linear $(m=2)$ resonance rather than the octupole $(m=4)$ resonance. At low density, these two stop bands almost disappear while ion losses at $Q_{0} \approx 3$ are still quite serious. The external-field-driven resonance condition for $P=9$,

$$
Q_{0} \approx 9\left(\frac{n}{m}\right)+C_{m} \Delta Q
$$

explains this observation. The rf potential of the LPT is not perfectly linear but contains some nonlinearity arising from mechanical imperfections such as slight electrode misalignments. According to Eq. (9), the lowest-order instability within the possible tune range $\left(0<Q_{0}<4.5\right)$ is the sextupole $(m=3)$ resonance at $Q_{0} \approx 3$. It is thus reasonable to conclude that ion losses at $Q_{0} \approx 3$ are induced largely by the third-order error field.

For completeness, we make some comments on the more general case where the two betatron tunes $Q_{0 x}$ and $Q_{0 y}$ are very different. We have already explored such situations in some previous works $[10,11,13]$. When the tune split is large, the horizontal and vertical instabilities are naturally separated (except for two-dimensional coupling resonance [10]). We then simply replace $Q_{0}$ and $\Delta Q$ in Eq. (7) by the horizontal (vertical) bare tune $Q_{0 x(0 y)}$ and tune shift $\Delta Q_{x(y)}$ to locate one-dimensional resonance lines on the so-called tune diagram. This means that each of the three instability regions in Fig. 4 involves at least two resonances of the same order excited simultaneously in the horizontal and vertical directions (cf. Fig. 7 in Sec. III B). If we increase or decrease $Q_{0 x}$ independently of $Q_{0 y}$, all three stop bands will eventually break up into at least two bands. In order to clarify the global resonance feature graphically, we need one more dimension; namely, the ion-loss distribution has to be plotted not as a function of $Q_{0}$ but on the tune diagram $[10,11,13]$. When the tune split is small, the standard linear transport theory based on the root-mean-squared (rms) envelope equations predicts the existence of the in-phase mode and out-of-phase mode at $\sigma_{0} \approx 90^{\circ}$ (corresponding to $Q_{0} \approx 2.25$ in Fig. 4) [6,7]. We have, however, observed no signature of the splitting of these linear eigenmodes in past S-POD experiments. An extensive study of the envelope equations by Lund and Bukh [26] has actually pointed out that the parameter regions within which the two modes lose stability largely overlap in a quadrupole doublet channel unlike in a solenoidal focusing channel. These envelope instabilities are thus not separable here.

\section{B. Excitation of half-integer stop bands}

We now disturb the ninefold symmetry of the lattice superperiod, switching on the perturbing pulses in Fig. 3(b). Equation (7) then gives the condition of coherent dipole resonance,

$$
Q_{0}=\frac{n}{2},
$$

and the condition of lattice-induced quadrupole resonance,

$$
Q_{0}-C_{2} \Delta Q=\frac{n}{2}
$$




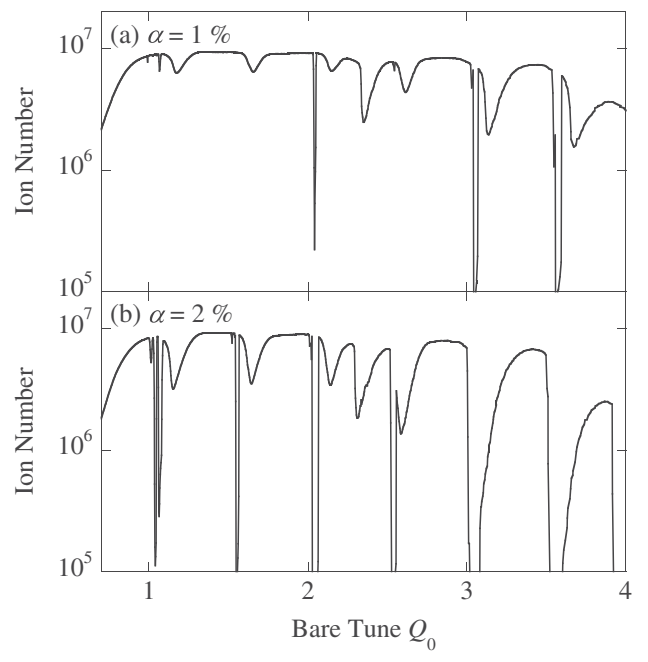

FIG. 5. Stop-band distributions in the presence of the superperiodic perturbation. The number of ${ }^{40} \mathrm{Ar}^{+}$ions surviving after $1 \mathrm{~ms}$ is plotted as a function of the bare tune $Q_{0}$ evaluated in disregard of the perturbing pulses. The actual horizontal and vertical tunes $\left(Q_{0 x}, Q_{0 y}\right)$ are slightly different from $Q_{0}$. The initial ion number is set roughly at $10^{7}$ in all measurements. The perturbation voltage relative to the sinusoidal focusing amplitude is chosen to be $\alpha=1 \%$ in the upper picture (a) and $2 \%$ in the lower picture (b).

Figure 5(a) shows a typical stop-band distribution after the excitation of the superperiodic perturbation in Fig. 3(b). In addition to the low-order stop bands already identified in Fig. 4, many new stop bands are created near or slightly above the bare tunes of $1,1.5,2,2.5,3$, and 3.5. We recognize that some of these new instability regions include two distinct stop bands. For instance, see the vicinity of $Q_{0}=2$. Very sharp ion losses have occurred almost exactly at $Q_{0}=2$ while a relatively wide stop band is also generated slightly above this tune. Similar ion-loss behavior can be seen at $Q_{0}=3$ and 3.5. The double stop-band structure in the vicinity of $Q_{0}=n / 2$ is much clearer in Fig. 5(b) where the perturbation has been made twice stronger. We can confirm the existence of sharp stop bands at $Q_{0}=1,1.5$, and 2.5 as well. The origin of these narrow stop bands must be the coherent dipole instability expected under the condition in Eq. (10). The wider stop band located slightly above each narrow band originates from the quadrupole resonance predicted by Eq. (11) [27].

The double band structure should not be confused with the splitting of a particular mode's stop band under the condition $Q_{0 x} \neq Q_{0 y}$. It is a result of overlapping of two essentially different modes near half-integer tunes. The unique nature of the coherent dipole resonance $(m=1)$, i.e., the very narrow band width and independency of charge density, enables us to distinguish it from the quadrupole resonance $(m=2)$ easily despite that both instabilities are located close to half-integer tunes. Note also that the periodic perturbation generated by the

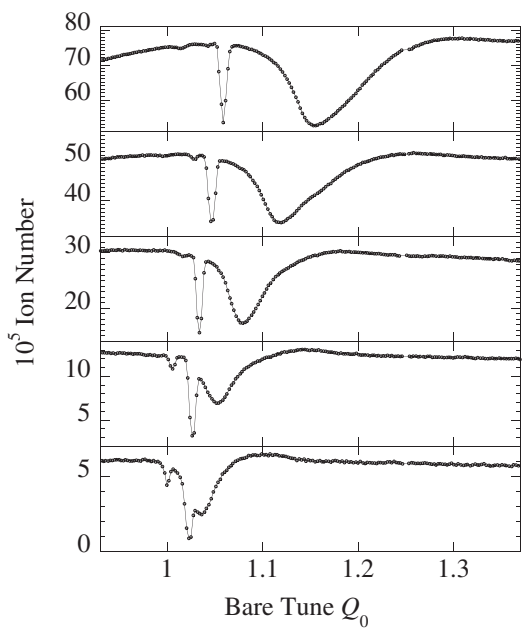

FIG. 6. Intensity dependence of the double stop-band structure near $Q_{0}=1$. The perturbation pulse height $V_{\mathrm{sp}}$ is the same as the case in Fig. 5(a); namely, $\alpha=1 \%$. The ion storage period is fixed at $1 \mathrm{~ms}$ in all experiments performed at different initial plasma densities.

electrode excitation pattern in Fig. 2(b) has quadrupolar symmetry as mentioned above. It cannot directly drive the breathing-mode oscillation.

Since the quadrupole resonance condition (11) contains the space-charge-induced tune shift $\Delta Q$, the locations of the wide stop bands vary depending on the ion density. The dipole resonance condition (10) is free from $\Delta Q$. The sharp stop bands are, therefore, supposed to stay at half-integer tunes regardless of the ion density. We can check this with the S-POD data in Fig. 6 where the behavior of the double band structure near $Q_{0}=1$ is depicted as an example. The wide quadrupole stop band has certainly shifted to the higher tune side as the initial ion number is raised. On the other hand, a slight shift was found for the sharp dipoleinduced dip, contrary to the theoretical expectation. The shift is much smaller than that of the quadrupole band but looks intensity dependent. According to careful PIC simulations, image charges on the electrode surfaces can be a source of this shift. A similar effect is often referred to as the Laslett tune shift in beam dynamics [28].

The result in Fig. 6 has also revealed the existence of another narrow dip next to the strong dipole stop band. The small dip is attributed to the second dipole resonance excited in the direction perpendicular to the other one. Such stop-band splitting naturally happens when $Q_{0 x} \neq Q_{0 y}$ [13]. The mechanism is quite simple as schematically outlined in Fig. 7. The perturbation pulse in Fig. 2(b) makes the horizontal and vertical tunes slightly different because it generates a quadrupole field. In the present experiment, we always applied a positive perturbing voltage to the horizontal electrodes, which somewhat enhances the focusing force in that direction. A series of ion-loss measurements are then performed along the broken line in Fig. 7. It is slightly shifted from the original 


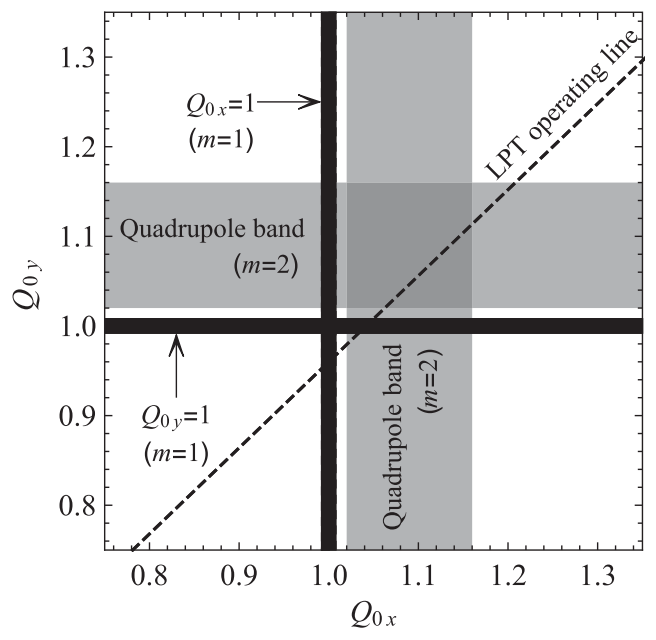

FIG. 7. Schematic drawing of the tune diagram in the vicinity of $Q_{0}=1$. The coherent dipole resonance occurs under the conditions $Q_{0 x(0 y)} \approx 1$ indicated by the thick horizontal and vertical lines. The hatched areas represent the stop bands within which the quadrupole mode becomes resonantly unstable. The ion-loss measurements in Fig. 6 have been performed along the broken line slightly shifted downward from $Q_{0 x}-Q_{0 y}=0$ due to the superperiodic perturbation. If we start the measurements from the low tune side, the horizontal dipole resonance $\left(Q_{0 x} \approx 1\right)$ is encountered first and then the vertical $\left(Q_{0 y} \approx 1\right)$. The horizontal and vertical stop bands of the quadrupole instability are too wide and, therefore, not separable in the present case where the perturbation-induced difference between $Q_{0 x}$ and $Q_{0 y}$ is small.

operating line $Q_{0 x}-Q_{0 y}=0$ on which the horizontal and vertical resonance bands intersect. As a result, we observe two sharp ion-loss regions corresponding to the conditions $Q_{0 x} \approx n / 2$ (horizontal dipole resonance) and $Q_{0 y} \approx n / 2$ (vertical dipole resonance). Similar stop-band splitting is expected also for the quadrupole resonance but not recognizable in the case of small tune difference because the quadrupole bands are too wide. In Fig. 6, the perturbation strength has been fixed at $\alpha=1 \%$. The resultant shifts of $Q_{0 x}$ and $Q_{0 y}$ from $Q_{0}$ are roughly $\pm 0.5 \%$.

The coherent dipole resonance observed here has nothing to do with error fields. Although it is possible to artificially excite the so-called "integer resonance" by applying transverse periodic kicks to the plasma [29], no such driving field was employed in this experiment (nor in the Vlasov theory [4] concluding the dipole-mode instability at half integers). A transverse dipole kick in Fig. 2(c) is used later in the next subsection, but it is just a single kick to make a small initial displacement of the bunch centroid. In principle, all kinds of parametric resonances can take place under the condition (7) if the initial particle distribution is deviated from the perfect stationary state finegrainedly matched to the external focusing potential. While highly nonlinear resonances are probably Landau damped or too weak to be visible in practice, some low-order instabilities can affect the beam quality leading to noticeable ion losses as demonstrated in Figs. 4 and 5. The seed of the coherent dipole instability is the initial deviation of the beam centroid from the ideal closed orbit of the machine. A finite transverse displacement of the bunch will constantly grow at half-integer tunes just like the linear betatron resonance of a single particle. This resonance is the lowest order and thus so strong that it causes serious ion losses once excited. Other collective resonances are a sort of self-restraint because the condition (7) depends on the tune shift $\Delta Q$; the instability gives rise to a gradual decrease in particle density or, in other words, a reduction of $\Delta Q$, which eventually moves the effective operating point out of the resonance band [30]. The independency of the dipole instability condition (10) on the space-chargeinduced tune shift implies that this resonance may be pretty severe at any density, but it also suggests the possibility of complete suppression of the dipole motion by controlling the initial beam position. In fact, the sharp stop bands at $Q_{0}=1,1.5$, and 2.5 in Fig. 5(a) are almost invisible somehow. One possible reason for this should be because the plasma centroid happens to be located very close to the center of the quadrupole focusing field. In that case, it takes the dipole mode a very long period to grow and yield ion losses.

\section{Time evolution}

Let us examine how the double-band structure evolves in time. In this subsection, we only pay attention to the region about $Q_{0}=2$ because other instability regions have an analogous feature. The typical time evolution of the band configuration is displayed in Fig. 8. We usually detect the

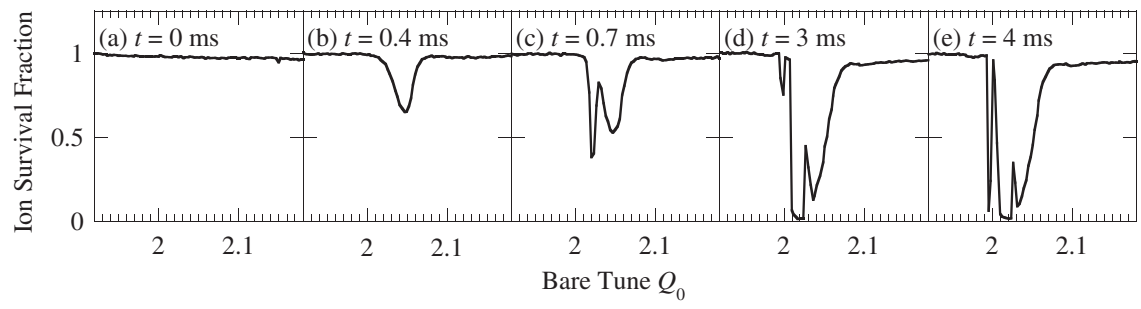

FIG. 8. Time evolution of the dipole and quadrupole instability bands near $Q_{0}=2$. The ordinate represents the number of surviving ions after a certain storage period, normalized with the initial ion number $\left(\approx 10^{6}\right.$ in this measurement). The perturbation strength has been fixed at $\alpha=1 \%$. 

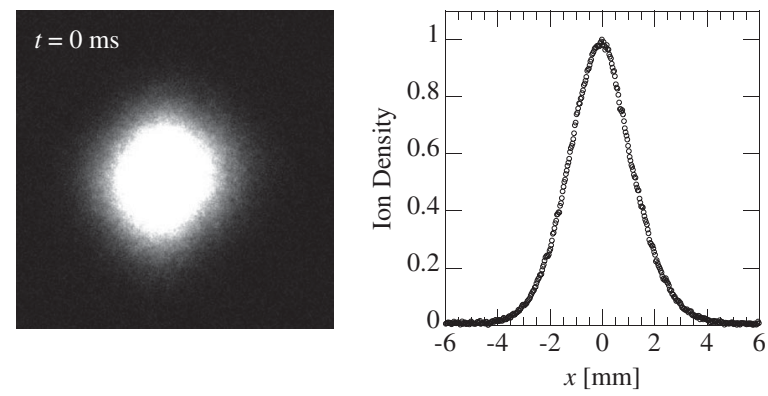

FIG. 9. Initial transverse profile of an ion bunch at $Q_{0}=1.99$. The right panel shows the density profile on the horizontal $x$ axis derived from the phosphor image in the left panel.

gradually growing instability of the quadrupole mode first, followed by the sudden birth of a sharp dipole band. Similarly to the result in Fig. 6, the second sharp instability band eventually appears just below $Q_{0}=2$. Considering the polarity of the quadrupole perturbation pulse, this second band should come from the horizontal dipole resonance while the first narrow band slightly above $Q_{0}=2$ from the vertical (cf. Fig. 7). This expectation has been verified in Figs. 9 and 10 that show the transverse plasma profiles on the phosphor screen. At the beginning, the plasma has roughly a rotationally symmetric profile at $Q_{0}=1.99$ (Fig. 9). After $2.55 \mathrm{~ms}$, the initial profile is remarkably changed as depicted in Fig. 10(a) because the operating point is in the middle of the left narrow instability band in Fig. 8(e). The transverse ion distribution looks expanded in the horizontal direction, which indicates that the bunch is oscillating horizontally. The direction of the bunch oscillation is vertical at $Q_{0}=2.02$ where the first narrow band is located. This is strong evidence that the two sharp ion losses around $Q_{0}=2$ are caused by the blowup (a) $Q_{0}=1.99$

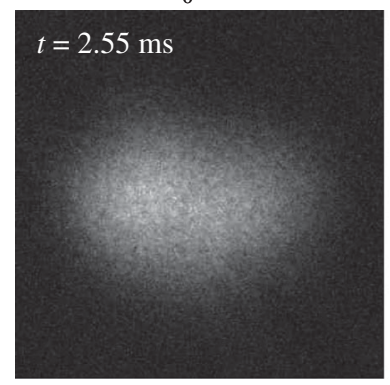

(b) $Q_{0}=2.02$

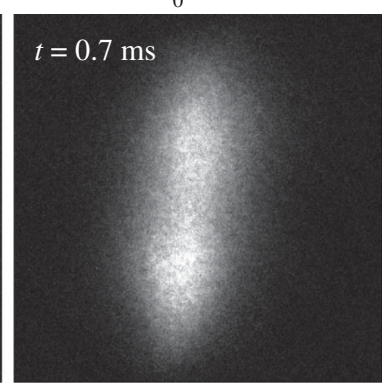

FIG. 10. Transverse profiles of ion bunches projected onto the phosphor screen. The left panel (a) and right panel (b) are the phosphor images measured after a $2.55-\mathrm{ms}$ storage of ions at $Q_{0}=1.99$ and after a $0.7-\mathrm{ms}$ storage of ions at $Q_{0}=2.02$, respectively. Sharp ion losses occur at these tunes, as indicated in Fig. 8. Note that individual ions hit the screen at different timings, depending on their axial positions and velocities when the potential barrier on the MCP side is removed for plasma extraction. The phosphor images shown here thus reflect the axial integrations of transversely oscillating ion distributions over a certain extraction period. of the coherent dipole oscillations in the horizontal and vertical directions [31].

Since the MCP is placed $10 \mathrm{~mm}$ away from the LPT exit, the plasma is radially expanded to some degree before reaching the MCP surface (while the overall profile is not affected). We did three dimensional simulations with our original code to evaluate the expansion factor. The estimated expansion rate was $130 \%$ under the experimental condition of Fig. 9. Since the rms extent of the transverse profile in Fig. 9 is $2.6 \mathrm{~mm}$, the plasma core before extraction should have the rms diameter of about $2 \mathrm{~mm}$, which is $20 \%$ of the LPT aperture. The core and tail certainly come closer to the electrode surfaces while executing the dipole oscillation, so it is not surprising even if we recognize the effect of image charges at high density. In a real circular machine, the safety factor is taken as large as possible to minimize beam losses due to halo formation and other unwanted effects. The stop-band shift due to image charges is, therefore, most likely small in general. For example, the RCS was designed to suppress the Laslett tune shift below 0.16 around the ring [32]. The shift per lattice period is below $\sim 0.05$, which looks at most comparable to what we found in Fig. 6. This suggests that the coherent dipole bands at RCS, if observable, should be located very close to half-integer tunes per lattice period even in the high-power operation.

It is informative to investigate if the coherent dipole instability is more enhanced by a larger initial displacement of the bunch centroid. For this purpose, we intentionally kicked the whole bunch once by applying an electric dipole field in Fig. 2(c). A pulse voltage of $1 \mu \mathrm{s}$ in width (FWHM) was given simultaneously to the horizontal pair of quadrupole rods right after a proper number of ions were accumulated in the LPT. The voltages on the two rods have an identical absolute height $V_{\text {kick }}$ but opposite signs to produce a dipole field around the LPT axis. Figure 11 shows the time evolution of ion losses within the three stop bands observed in Fig. 8. We tried a couple of different $V_{\text {kick }}$ 's to change the size of the initial bunch displacement. The operating tunes in the left and middle panels are adjusted, respectively, to the horizontal and vertical dipole bands sitting slightly below and above $Q_{0}=2$. As for the quadrupole instability (right panel), the stop-band location moves significantly within $4 \mathrm{~ms}$, responding to the plasma density reduction. It is evident from the left panel (a) that the dipole instability is activated earlier in the horizontal direction as we strengthen the initial kick. Since the intentional kick is applied only horizontally, it has no effect on the vertical dipole motion as is obvious from the middle panel (b). The right panel (c) makes it clear that the quadrupole instability is independent of $V_{\text {kick}}$.

We also checked the intensity dependence of the dipole instability. If the resonance condition in Eq. (10) is valid, the time evolution of ion losses within a sharp stop band must be insensitive to the number of ions initially stored in 


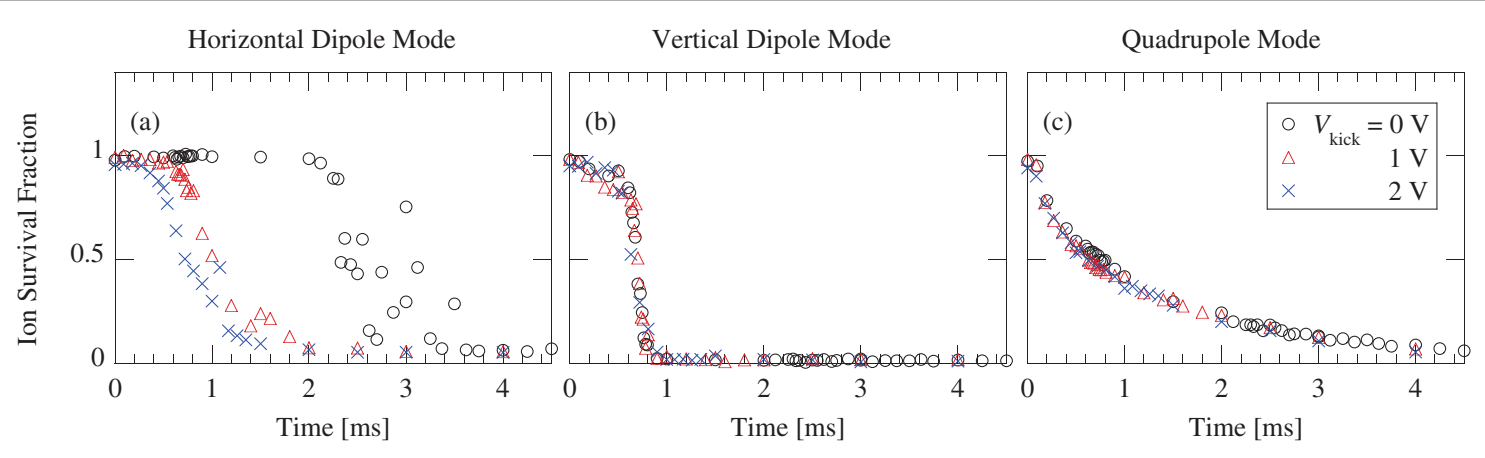

FIG. 11. Effect of an initial transverse displacement of the bunch centroid on the dipole and quadrupole instabilities near $Q_{0}=2$. An ion bunch in the LPT has been kicked once at $t=0[\mathrm{~ms}]$ in the horizontal direction by a dipole electric pulse in Fig. 2(c). Three symbols correspond to three different kick voltages, namely, $V_{\text {kick }}=0$ [V] (circle), 1 [V] (triangle), and 2 [V] (cross). The number of ions initially stored in the LPT is about $10^{6}$ in all three cases. The operating tune in each panel is adjusted to the point at which the ion loss rate becomes maximum within a particular stop band. For the dipole instability, the adjusted tune stays close to (a) $Q_{0}=1.99$ or (b) $Q_{0}=2.02$ throughout the ion-loss measurements. In case (c), the operating tune has been reduced from $\sim 2.05$ down to $\sim 2.03$ in $4 \mathrm{~ms}$ because the quadrupole stop band moves to the lower tune side with a gradual decrease in the ion density.

the LPT. The fractions of surviving ions measured at different initial bunch intensities are plotted in Fig. 12. The operating tune is adjusted to the horizontal dipole band located slightly below $Q_{0}=2$. No apparent correlation between ion losses and density is seen as expected.

The seed of the quadrupole and other higher-order parametric instabilities $(m \geq 2)$ is a distortion of the initial plasma profile from the ideal stationary distribution corresponding to a self-consistent solution to the VlasovPoisson equations. As explained in Sec. II, we can generate in S-POD a quasistationary plasma well matched to the external AG potential. The initial distortion is considered to be quite weak. We can thus reproduce similar ion-loss behavior whenever the same set of experimental parameters is chosen. This argument, however, seems inapplicable to

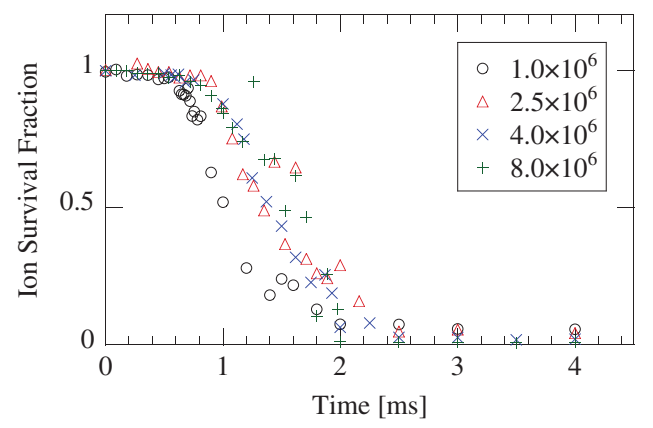

FIG. 12. Intensity dependence of ion-loss behavior under coherent dipole resonance. The time evolution of the fraction of surviving ions was measured with the MCP, starting from several different plasma densities. The initial ion numbers in the LPT are $1.0 \times 10^{6}$ (circle), $2.5 \times 10^{6}$ (triangle), $4.0 \times 10^{6}$ (cross), and $8.0 \times 10^{6}$ (plus). The horizontal kick voltage of $V_{\text {kick }}=1[\mathrm{~V}]$ has been used in all four cases. The operating point is the same as that in Fig. 11(a), namely, $Q_{0} \approx 1.99$ where the ion loss rate becomes maximum due to the horizontal coherent dipole instability. the coherent dipole resonance $(m=1)$. The dipole mode is unique compared to the other modes as it has nothing to do with the ion distribution. The growth rate is even independent of ion density. What matters is only the distance between the plasma centroid and trap axis. As already demonstrated, this parameter can be controlled by switching on a pulse dipole voltage $V_{\text {kick}}$. In case we do not use this intentional dipole kick, the possible initial displacement of the plasma centroid from the axis is determined by some vague factors including systematic noises, statistics, etc., peculiar to the S-POD experiment. See, for instance, one of the two dipole stop bands in Fig. 6 (the small dip at $Q_{0}=1$ ). It shrinks as the number of initially stored ions becomes larger. We suspect that this is a sort of statistical effect; namely, the possible initial shift of the centroid is statistically reduced as we increase the ion number. The same tendency has been confirmed in another S-POD experiment assuming the lattice symmetry of the Proton Synchrotron at CERN. We then wonder why the other dipole stop band in Fig. 6 (the very sharp ion losses a bit above $Q_{0}=1$ ) remains prominent even at high density. This is mostly due to technical limitations in controlling the rf waveforms by our power generating device. At present, even after careful rf phase and amplitude adjustments, we still have some error that gives rise to a weak vertical dipole field during the ion accumulation process. As long as the current setup of the rf system is maintained, the plasma initially has a constant vertical shift much greater than the possible statistical displacement. This is why we always see the vertical dipole oscillation growing more rapidly than the horizontal one in the absence of the initial kick (cf. Figs. 8 and 11).

\section{NUMERICAL SIMULATION}

We carried out systematic multiparticle simulations to support our conclusions in Sec. III. The PIC code WARP 


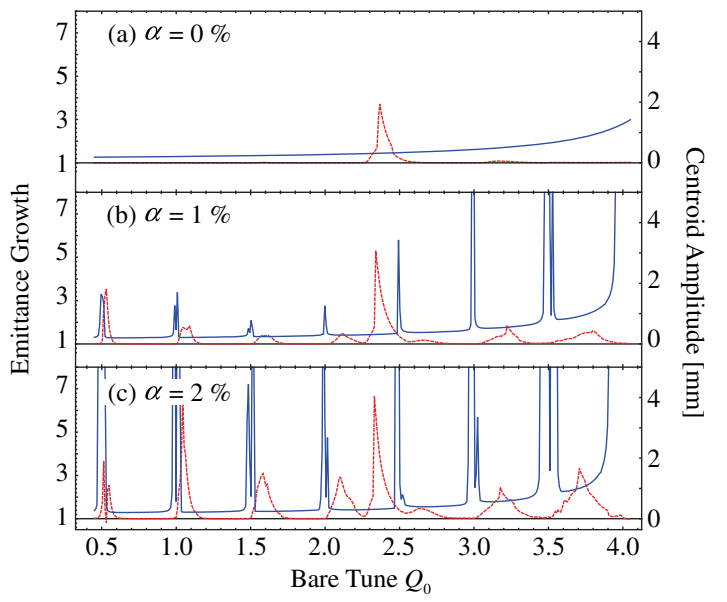

FIG. 13. WARP simulation results assuming the AG lattice waveforms in Fig. 3. The rate of rms emittance growth (red broken line) and the position of the plasma centroid (blue solid line) after a storage period of $0.5 \mathrm{~ms}$ are numerically evaluated at different operating tunes. The plasma centroid is displaced initially by $0.1 \mathrm{~mm}$ in both transverse directions to activate the coherent dipole oscillation. The tune depression has been fixed at 0.9 in all three cases where the perturbation strength parameter $\alpha$ is chosen to be (a) $0 \%$, (b) $1 \%$, and (c) $2 \%$.

[33] was employed for this purpose. Figure 13 shows WARP simulation results based on the experimental conditions in Figs. 4 and 5. Recalling the measured plasma profile in Fig. 9, we have assumed the Gaussian-type initial distribution whose tune depression is adjusted to 0.9 . The excellent macroparticle distribution generator of the WARP code enables us to achieve not the crude rms matching but a sophisticated self-consistent matching including the Debye screening effect [34]. In Fig. 13, the transverse rms emittance after a storage period of $0.5 \mathrm{~ms}$, normalized with its initial value, is drawn in red as a function of operating tune. In the upper panel (a) where the superperiodic perturbation is off, we observe a clear peak rising in the region $2.25 \leq Q_{0} \leq 2.5$. This is the self-field-induced quadrupole $(m=2)$ stop band shifted upward from $Q_{0}=9 / 4$. The existence of this severe stop band has been confirmed by S-POD experiments in Figs. 4 and 5. Unlike the experimental data in Fig. 4, the thirdorder $(m=3)$ instability at $Q_{0} \approx 3$ is very weak here because we did not consider nonlinear imperfection fields in these simulations. The external driving force assumed in Fig. 13 is perfectly linear. Ion losses at $Q_{0} \approx 3$ can considerably be enhanced by introducing the effect of electrode misalignments in WARP simulations [12]. The middle and lower panels show what happens when the superperiodic perturbation in Fig. 3(b) is added to the sinusoidal focusing wave. Many additional instability bands have appeared near the particular tunes derived from the quadrupole resonance condition in Eq. (11). The coherent dipole resonance under the condition (10) gives rise to no emittance growth because the whole bunch simply executes a transverse oscillation with no significant deformation of the ion distribution (cf. Fig. 15). We, therefore, followed the transverse position of the bunch centroid to see whether the dipole motion is stable or growing. The blue solid line in each picture indicates the maximum amplitude of the centroid oscillation after $0.5 \mathrm{~ms}$. In these simulations, we put the bunch slightly off axis at the beginning to activate the dipole motion. Otherwise, no dipole instability is excited within a reasonable computing time. We confirm the rapid growth of the transverse oscillation amplitude at $Q_{0}=n / 2$, which shall be detected as serious ion losses in S-POD experiments. Both dipole and quadrupole instabilities have become more prominent as the superperiodic perturbation is strengthened. As expected, two sharp peaks are formed side by side about $Q_{0}=n / 2$, one of which is due to the horizontal dipole motion and the other to the vertical. These numerical observations are in good agreement with the experimental data in Fig. 5. We have also done similar simulations with the superperiodic perturbation of a different pulse width (FWHM of $0.5 \mu \mathrm{s}$ instead of $1.0 \mu \mathrm{s}$ ), confirming no essential change in the stop-band distribution [35].

The coherent dipole oscillations about $Q_{0}=2$ are plotted in Fig. 14. The oscillation amplitude grows horizontally at $Q_{0}=1.9962$ and vertically at $Q_{0}=2.0043$,
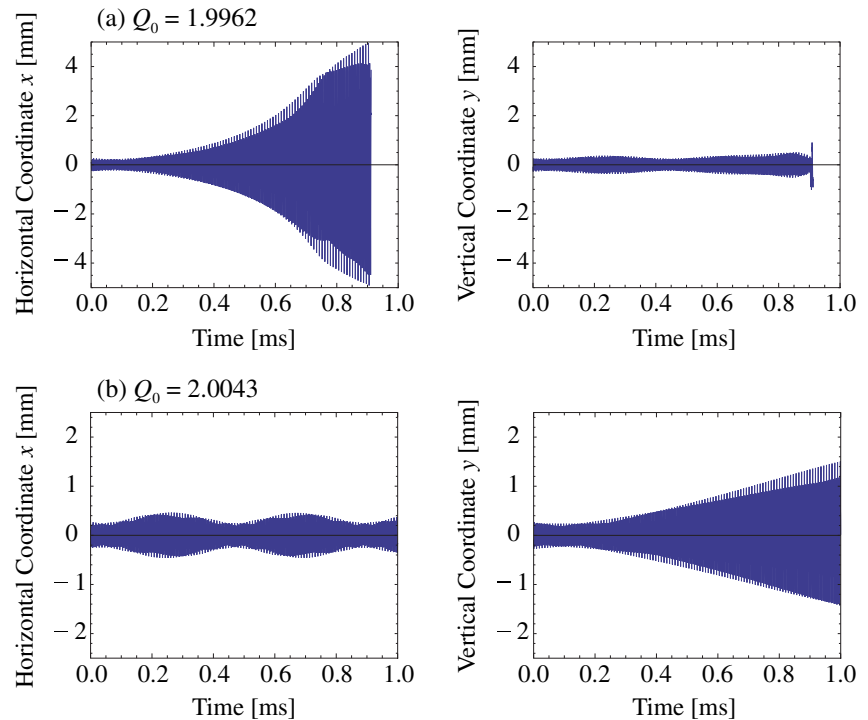

FIG. 14. Transverse oscillations of the bunch centroid at slightly different operating points about $Q_{0}=2$. The perturbation strength $\alpha$ is $1 \%$ in both examples. The tune depression is fixed at 0.9. The effect of the quadrupole rods (perfect conductor) is included in these simulations. The bunch centroid has been shifted by $0.1 \mathrm{~mm}$ at the beginning to excite the coherent dipole motion. Without this intentional initial shift, the bunch stays on axis for a very long period. Macroparticles are removed when their transverse distances from the axis exceed the LPT aperture size, i.e., $5 \mathrm{~mm}$ in radius. In the upper case (a), all particles have been lost at $t \approx 0.9$ [ms] due to a large horizontal dipole oscillation. 

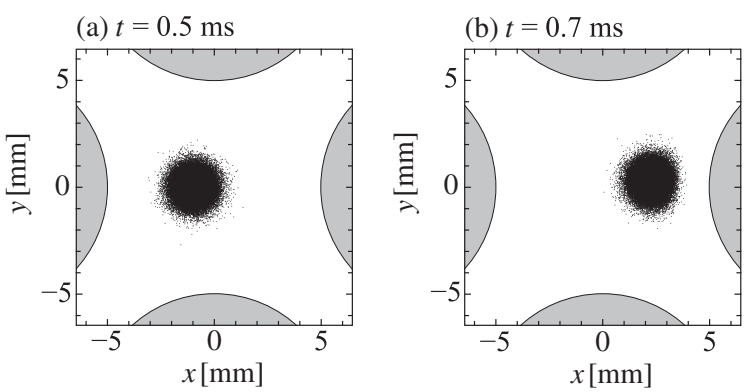

FIG. 15. Transverse profiles of an ion bunch at (a) $t=0.5[\mathrm{~ms}]$ and (b) $t=0.7[\mathrm{~ms}]$. The simulation parameters are identical to those assumed in Fig. 14(a). The four hatched areas indicate the quadrupole rods.

which is consistent with the bunch profile measurements in Fig. 10. For reference, we show in Fig. 15 the crosssectional views of an ion bunch after 0.5 and $0.7 \mathrm{~ms}$ when $Q_{0}=1.9962$. The whole bunch is obviously executing a rigid dipole oscillation in the horizontal direction. A weak asymmetric distortion from the circular bunch profile in the right panel is caused by a quadrupole electrode. Results of ion-loss simulations with $Q_{0}=1.9962$ are summarized in Fig. 16. In this figure as well as in Figs. 14 and 15, four perfectly conducting rods placed $5 \mathrm{~mm}$ away from the LPT axis have been taken into account when we solve the Poisson equation at every numerical integration step. A macroparticle is judged lost once its transverse oscillation amplitude exceeds the aperture size of the LPT. Since the vertical dipole motion is stable at $Q_{0}=1.9962$, we only horizontally displaced the bunch by $\Delta x$ at the start of each simulation in Fig. 16. (The result is unchanged even if a slight vertical displacement is given to the bunch initially.) Naturally, no particle losses take place when $\Delta x=0$. Stored ions begin to be lost earlier with a larger initial displacement of the bunch centroid. This observation qualitatively agrees with the S-POD data in Fig. 11(a).

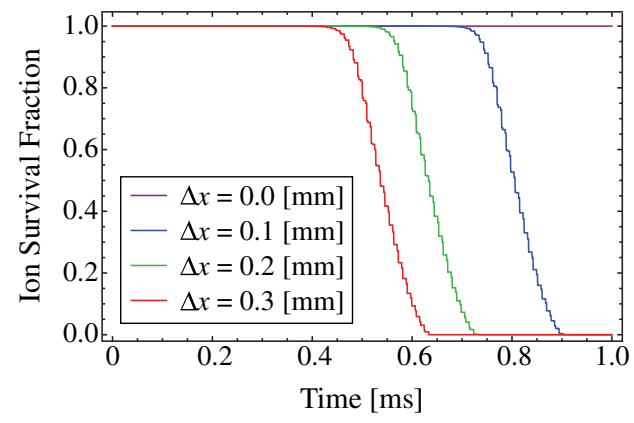

FIG. 16. Ion loss simulations by the WARP code. The simulation parameters are identical to those assumed in Fig. 14(a) except for the size of the initial bunch displacement. The bunch is only horizontally displaced by $0,0.1,0.2$, and $0.3 \mathrm{~mm}$ at $t=0$ [ms].

\section{SUMMARY}

The novel experimental system S-POD was employed to demonstrate that two different types of collective instability mechanisms can exist near every half-integer tune per lattice superperiod of a high-intensity ring. We controlled the waveform of the AG focusing function $K(t)$ in Eq. (6) to emulate the approximate lattice condition of a specific machine. The present work treated, without loss of generality, the case where each lattice superperiod includes nine FODO cells $\left(N_{\text {cell }}=9\right)$. We obtained convincing experimental evidence that the coherent instability of the dipole mode $(m=1)$ can be excited at half-integer tunes overlapping with the well-known quadrupole resonance $(m=2)$. It has been confirmed that the coherent dipole resonance has a very narrow band width and is insensitive to the bunch density. Aside from a slight shift possibly due to image charges on the metallic electrodes, each dipole stop band stays close to a half-integer tune. In contrast, the quadrupole resonance yields a wider stop band that moves to the higher tune side as the bunch becomes denser. These experimental observations with S-POD are consistent not only with theoretical expectations but also with numerical simulations by a PIC code.

Needless to say, the dipole instability is excited in either the horizontal or vertical direction (or both directions simultaneously) depending on the machine operating point, so is the quadrupole instability. The present experiment was performed under the condition where the bare tunes in the two transverse directions are nearly equal. The horizontal and vertical instability bands are, therefore, expected to largely overlap each other as illustrated in Fig. 7. Nevertheless, the dipole stop bands of the two directions were separable because of the very narrow band width.

The amplitude growth of the dipole mode occurs in the same manner as the simple half-integer resonance of a single particle. The seed of the coherent dipole instability is a finite deviation of the bunch centroid from the ideal position. The detail of the charge distribution does not matter. As experimentally verified with S-POD, this type of resonance is very severe leading to the loss of a whole bunch once excited. The dipole resonance can, however, be suppressed by setting the bunch centroid exactly on the design closed orbit, unlike the coherent quadrupole resonance seeded by a finite distortion of the charge distribution from the perfect stationary state. (The precise control of the particle distribution function in phase space is practically impossible.) As shown in Sec. III experimentally and in Sec. IV numerically, the dipole oscillation starts to grow earlier when the initial deviation of the bunch centroid from the LPT axis (in other words, from the ideal beam orbit) is greater. Such an error in bunch positioning more or less takes place at beam injection into a circular machine. Very narrow coherent dipole stop bands may thus be observed at half-integer tunes in practice before careful beam orbit correction. 


\section{ACKNOWLEDGMENTS}

The authors would like to thank Dr. Shinji Machida for valuable comments on the present experiment. One of the authors (M. O.) is indebted to Mr. Okano for his kind assistance in operating the S-POD system. This work is supported in part by JSPS KAKENHI Grant No. $15 \mathrm{H} 03662$.

[1] F. Sacherer, Transverse Space-Charge Effects In Circular Accelerators, Ph.D. thesis, Lawrence Radiation Laboratory, Berkeley, California [Report No. UCRL-18454, 1968].

[2] R. Baartman, Betatron Resonances with Space Charge, Proceedings, Space Charge Physics in High Intensity Hadron Rings: Shelter Island, New York, 1998, AIP Conf. Proc. 448, 56 (1998).

[3] $\Delta \nu$ can uniquely be determined in the Sacherer's Vlasov theory [1] that has assumed a uniform particle distribution in real space. Other nonlinear distributions have a finite tune spread depending on the beam density. $\Delta \nu$ is then often replaced by the root-mean-squared average $\Delta \bar{\nu}$ of incoherent tune shifts over all particles.

[4] H. Okamoto and K. Yokoya, Parametric resonances in intense one-dimensional beams propagating through a periodic focusing channel, Nucl. Instrum. Methods Phys. Res., Sect. A 482, 51 (2002).

[5] J. Struckmeier, J. Klabunde, and M. Reiser, On The Stability And Emittance Growth Of Different Particle Phase-Space Distributions In A Long Magnetic Quadrupole Channel, Part. Accel. 15, 47 (1984).

[6] M. Reiser, Theory and Design of Charged Particle Beams (John Wiley \& Sons, New York, 2008), and references therein.

[7] I. Hofmann, L. J. Laslett, L. Smith, and I. Haber, Stability Of The Kapchinskij-Vladimirskij (K-V) Distribution In Long Periodic Transport Systems, Part. Accel. 13, 145 (1983).

[8] A. V. Fedotov, I. Hofmann, R. L. Gluckstern, and H. Okamoto, Parametric collective resonances and spacecharge limit in high-intensity rings, Phys. Rev. ST Accel. Beams 6, 094201 (2003).

[9] Coherent resonances driven by nonlinear imperfections have been studied analytically in Sec. VI of Ref. [4]. External error fields enhance beam instability under the condition (44) that is equivalent to Eq. (2) in the present paper.

[10] H. Okamoto, K. Ito, K. Fukushima, and T. Okano, Recent Results From The S-Pod Trap Systems On The Stability Of Intense Hadron Beams, in Proceedings of the 54th ICFA Advanced Beam Dynamics Workshop on High-Intensity and High-Brightness Hadron Beams (HB2014) Michigan, 2015, TUO2LRO3 (JACoW, 2015), pp. 178-182.

[11] H. Okamoto et al., Beam Dynamics Studies With Non-Neutral Plasma Traps, in Proceedings of the 5th International Particle Accelerator Conference, Dresden, Germany, 2014, FRXAA01 (JACoW, 2014), pp. 4052-4056.

[12] H. Takeuchi, K. Fukushima, K. Ito, K. Moriya, H. Okamoto, and H. Sugimoto, Experimental study of resonance crossing with a Paul trap, Phys. Rev. ST Accel. Beams 15, 074201 (2012).

[13] K. Fukushima, K. Ito, H. Okamoto, S. Yamaguchi, K. Moriya, H. Higaki, T. Okano, and S. M. Lund, Experimental verification of resonance instability bands in quadrupole doublet focusing channels, Nucl. Instrum. Methods Phys. Res., Sect. A 733, 18 (2014).

[14] H. Okamoto, M. Endo, K. Fukushima, H. Higaki, K. Ito, K. Moriya, S. Yamaguchi, and S. M. Lund, Experimental simulation of beam propagation over long path lengths using radio-frequency and magnetic traps, Nucl. Instrum. Methods Phys. Res., Sect. A 733, 119 (2014).

[15] The Sacherer theory in Ref. [1] concludes $C_{1}=0$, while the Okamoto-Yokoya theory in Ref. [4] gives a small but finite value to $C_{1}$ due to the approximation introduced for an estimation of the tune spread $\Delta \nu$ in a waterbag beam. Since the rigid dipole oscillation should be independent of the beam density, we expect $C_{1}$ to be exactly zero (as long as the effect from image charges on the beam environment is negligible).

[16] H. Okamoto, Y. Wada, and R. Takai, Radio-frequency quadrupole trap as a tool for experimental beam physics, Nucl. Instrum. Methods Phys. Res., Sect. A 485, 244 (2002).

[17] R. Takai, H. Enokizono, K. Ito, Y. Mizuno, K. Okabe, and H. Okamoto, Development of a compact plasma trap for experimental beam physics, Jpn. J. Appl. Phys. 45, 5332 (2006).

[18] H. Okamoto and H. Tanaka, Proposed experiment for the study of beam halo formation, Nucl. Instrum. Methods Phys. Res., Sect. A 437, 178 (1999).

[19] P. K. Ghosh, Ion Traps (Oxford Science, Oxford, 1995).

[20] S. Sheehy and D. Kelliher (private communication).

[21] R. C. Davidson, H. Qin, and G. Shvets, A Paul trap configuration to simulate intense non-neutral beam propagation over large distances through a periodic focusing quadrupole magnetic field, Phys. Plasmas 7, 1020 (2000).

[22] E. P. Gilson, R. C. Davidson, M. Dorf, P. C. Efthimion, R. Majeski, M. Chung, M. S. Gutierrez, and A. N. Kabcenell, Studies of emittance growth and halo particle production in intense charged particle beams using the Paul Trap Simulator Experiment, Phys. Plasmas 17, 056707 (2010).

[23] E. P. Gilson, R. C. Davidson, P. C. Efthimion, R. Majeski, E. A. Startsev, H. Wang, S. Koppell, and M. Talley, Excitation of transverse dipole and quadrupole modes in a pure ion plasma in a linear Paul trap to study collective processes in intense beams, Phys. Plasmas 20, 055706 (2013).

[24] H. Hotchi et al., Beam commissioning and operation of the Japan Proton Accelerator Research Complex 3-GeV rapid cycling synchrotron, Prog. Theor. Exp. Phys. 2012, 02B003 (2012).

[25] Fifty years of the CERN Proton Synchrotron, edited by S. Gilardoni and D. Manglunki, Report No. CERN-2011-004, 2011.

[26] S. M. Lund and B. Bukh, Stability properties of the transverse envelope equations describing intense ion beam transport, Phys. Rev. ST Accel. Beams 7, 024801 (2004).

[27] The self-field-driven quadrupole resonance bands near $Q_{0} \approx n / 4$ are hardly observable at this intensity except 
for the one at $Q_{0} \approx 9 / 4(n=P)$. To enhance all of them, we must not only increase the ion density but also more strongly distort the ninefold lattice symmetry.

[28] L. J. Laslett, On intensity limitations imposed by transverse space-charge effects in circular particle accelerators, BNL Report No. 7534, 1963, pp. 325-367.

[29] K. Moriya, K. Fukushima, K. Ito, T. Okano, H. Okamoto, S. L. Sheehy, D. J. Kelliher, S. Machida, and C. R. Prior, Experimental study of integer resonance crossing in a nonscaling fixed field alternating gradient accelerator with a Paul ion trap, Phys. Rev. ST Accel. Beams 18, 034001 (2015).

[30] This self-restraint mechanism explains why the self-fielddriven linear resonance (e.g., the stop band at $Q_{0} \approx 2.25$ in Fig. 4) tends to induce more ion losses on the lower tune side of a band. According to Eq. (11), the stop band gradually moves leftwards as $\Delta Q$ is reduced by the instability. The plasma thus stays within the stop band for a longer period when the operating bare tune $Q_{0}$ is lower.

[31] The wide stop band of the quadrupole mode in Fig. 8 involves two overlapping instabilities in the horizontal and vertical directions (see Fig. 7). They are not separable unless the tune split is sufficiently large. When the difference between $Q_{0 x}$ and $Q_{0 y}$ is small, the plasma blows simultaneously in both transverse directions on the quadrupole resonance. The transverse image on the phosphor screen then expands radially. In order to obtain detailed information of various oscillation modes experimentally, we have to probe the phase space.

[32] Accelerator Technical Design Report for High-Intensity Proton Accelerator Facility Project, J-PARC, edited by Y. Yamazaki, Report No. JAERI-Tech 2003-044, KEK Report2002-13, 2003.

[33] D. P. Grote, A. Friedman, G. Craig, I. Haber, and W. Sharp, Progress toward source-to-target simulation, Nucl. Instrum. Methods Phys. Res., Sect. A 464, 563 (2001).

[34] S. M. Lund, T. Kikuchi, and R. C. Davidson, Generation of initial kinetic distributions for simulation of long-pulse charged particle beams with high space-charge intensity, Phys. Rev. ST Accel. Beams 12, 114801 (2009).

[35] Note that the amplitudes of the driving Fourier harmonics vary depending on the perturbation pulse shape. The change of the pulse width, therefore, results in somewhat different growth rates of resonances, but the global resonance picture remains unchanged. 